\title{
P15 Kita-Ernährung auf dem Prüfstand
}

\author{
Susanne Kunz • Simone Hoesl • Jörg Klewer
}

Online publiziert: 30. November 2011

(C) Springer-Verlag 2011

Ergebnisse des Bundesministeriums für Ernährung, Landwirtschaft und Verbraucherschutz, der KiGGS-Studie, der Verbraucherzentrale und der Deutschen Gesellschaft für Ernährung sowie Daten des Gesundheitsamtes Zwickau zeigten einen Handlungsbedarf. Ziel dieser Studie war die Untersuchung der Verpflegungssituation von Kindern in Kindertageseinrichtungen mit der Kernfrage, ob es einen erklärbaren Zusammenhang der Ernährungsqualität der Einrichtungen und den Trägern der Einrichtungen sowie weiteren Einflussfaktoren gibt.

Von 174 Kindertageseinrichtungen nahmen 150 an der schriftlichen Befragung zur Ernährung und Speisenversorgung der Kinder teil (Teilnahmequote: $86 \%$ ).

Die theoretisch zu erwartenden Unterschiede der Verpflegungssituation in Bezug auf die empfohlenen Qualitätsstandards konnten in den Ergebnissen anhand relativer Häufigkeiten nachgewiesen werden. Der Schwerpunkt war die Mittagsverpflegung, die bei $82 \%$ der Einrichtungen extern geliefert wird. Die Möglichkeit der Speisenplanbeeinflussung wird von $80 \%$ der Erzieher genutzt. Die Gesamtzufriedenheit mit den Mahlzeiten liegt bei den Kindern bei ca. $70 \%$ und 58,7\% aller Einrichtungen wünschen sich eine Zusammenarbeit zur Verbesserung der Verpflegungssituation. Die Ernährungsqualität wurde mit einer linearen Regression untersucht, wobei die Teilnahme der Erzieher am Mittagessen und deren Speisenplanbeeinflussung einen direkten Effekt ausübte.

S. Kunz $(\bowtie) \cdot$ J. Klewer

Fakultät für Gesundheits- und Pflegewissenschaften,

Westsächsische Hochschule Zwickau, Zwickau, Deutschland

S. Hoesl

Gesundheitsamt Zwickau, Zwickau, Deutschland
Diese Daten dienen als Grundlage zur Verbesserung der Verpflegungssituation. Es sollte nachhaltig eine qualitativ ausgewogene und vollwertige Verpflegung der Kinder in den Kitas des Landkreises Zwickau geben, welche als Basis die „Qualitätsstandards für die Verpflegung in Tageseinrichtungen für Kinder" umfasst und ein Prozess der Verpflegung schafft, der eine begleitende Ernährungskommunikation beinhaltet, an dem alle Beteiligten teilhaben können. 